\title{
CORRECTION
}

\section{Correction to: Education to save lives: C19SPACE, the COVID19 Skills PrepAration CoursE}

Stefan J. Schaller ${ }^{1,2,3}$ (D), Johannes Mellinghoff ${ }^{1,4}$ (1) and Maurizio Cecconi ${ }^{1,5,6^{*}}$ (D) on behalf of the C19_Space Taskforce members, ESICM

๑ 2022 Springer-Verlag GmbH Germany, part of Springer Nature

\section{Correction to:}

Intensive Care Med (2022) 48:227-230 https://doi.org/10.1007/s00134-021-06591-z

In this article the author name Mellinghoff was incorrectly written as Mellinhgoff. The original article has been corrected.

\section{Author details}

1 European Society of Intensive Care Medicine (ESICM), Bruxelles, Belgium. 2 Department of Anesthesiology and Operative Intensive Care Medicine, Charité - Universitätsmedizin Berlin, Corporate Member of Freie Universität Berlin, Humboldt-Universität zu Berlin, Berlin Institute of Health, Berlin, Germany. 3 Department of Anesthesiology and Intensive Care, School of Medicine, Technical University of Munich, Klinikum rechts der Isar, Munich, Germany. 4 School of Sports and Health Sciences, University of Brighton, Brighton, UK. 5 Department of Biomedical Sciences, Humanitas University, Pieve Emanuele, Milan, Italy. 6 Department of Anesthesia and Intensive Care Units, IRCCS Humanitas Research Hospital, Rozzano, Milan, Italy.

\section{Publisher's Note}

Springer Nature remains neutral with regard to jurisdictional claims in published maps and institutional affiliations.

Published: 14 February 2022 